Appl. Phys. B 51, 49-51 (1990).

\title{
CARS-Background Suppression by Phase-Controlled Nonlinear Interferometry
}

\author{
G. Marowsky and G. Lüpke \\ Max-Planck-Institut für biophysikalische Chemie, Abteilung Laserphysik, Am Fassberg, D-3400 Göttingen, \\ Fed. Rep. Germany
}

Received 22 January 1990/Accepted 29 March 1990

\begin{abstract}
We have developed a new technique for efficient suppression of the nonresonant background in coherent anti-Stokes Raman scattering (CARS). The advantage of this technique, which is based upon phase-controlled superposition of an appropriately selected reference signal, is presented in theory and demonstrated experimentally by the example of the $S(3)$ (pure rotational) transition of molecular hydrogen.
\end{abstract}

PACS: 42.65

Since its first demonstration by Maker and Terhune [1] coherent anti-Stokes Raman scattering (CARS) has been developed into a powerful analytic tool for the spectroscopic determination of various physico-chemical quantities: remote and non-intrusive temperature and concentration analysis is a typical example. An up to date survey is provided by $[2,3]$ and references therein. Even laser gain has been measured recently by CARS [4] by monitoring the laser operation dependent $Q$-branch manifold of molecular nitrogen. Trace analysis of minor constituents of multi-component mixtures has been so far limited by the fact that the resonant signal merges upon decrease of concentration into the nonresonant signal contribution ("nonresonant noise"). Various polarization techniques have been developed in the past to suppress the nonresonant background contribution [5-7]. They all have in common that they improve the signal-to-noise ratio but simultaneously also lower the strength of the resonant signal. It is the purpose of this report to show that by application of phase-controlled nonlinear interferometry [8] a resonant CARS-signal can be recouped from the background noise without any loss in its strength.

\section{Theory}

The basic idea of background cancellation by nonlinear interferometry was briefly mentioned for third-order processes in [8] and is actually a consequence of experimental results obtained with phasesensitive background studies in second-order nonlinear effects, such as second-harmonic generation (SHG) [9]. The amplitude of a CARS-signal can be described in terms of a respective third-order susceptibility $\chi^{(3)}$ given by the expression

$\chi^{(3)}=\chi_{\mathrm{NR}}^{(3)}+\chi^{(3)}(\Delta \omega)=\chi_{\mathrm{NR}}^{(3)}+\frac{\tilde{\chi}^{(3)}}{\Delta \omega-i \Gamma}$.

The quantity $\chi_{\mathrm{NR}}^{(3)}$ denotes the nonresonant contribution and $\chi^{(3)}(\Delta \omega)$ a complex Lorentzian with $\chi^{(3)}$ its amplitude, $\Delta \omega=\omega_{\mathrm{R}}-\left(\omega_{\mathrm{p}}-\omega_{\mathrm{s}}\right)$ the frequency difference between the incident beams of frequency $\omega_{p}$ (pump beam) and $\omega_{\mathrm{s}}$ (Stokes beam) and a Raman resonance of frequency $\omega_{\mathrm{R}}$, and $\Gamma$ the linewidth of the particular transition. Figure 1 displays the phase-dependent addition of $\chi_{\mathrm{NR}}^{(3)}$ and $\chi^{(3)}(\Delta \omega)$ in the complex plane. According to this figure background suppression can be achieved by addition of another nonresonant signal of opposite sign leading to a pure Lorentzian with an amplitude of the initial resonant signal. To facilitate a comparison between the experimental results and the magnitude of $\chi_{\mathrm{NR}}^{(3)}$ and $\chi^{(3)}(\Delta \omega)$ according to (1), schematic diagrams of Fig. 1 are shown as insets in the subsequent figures displaying actual CARS-spectra.

Phase-sensitive measurements require an a priori knowledge of the coherence length $L_{\mathrm{c}}=2 \pi / \Delta k$, with $\Delta k$ the difference in wave

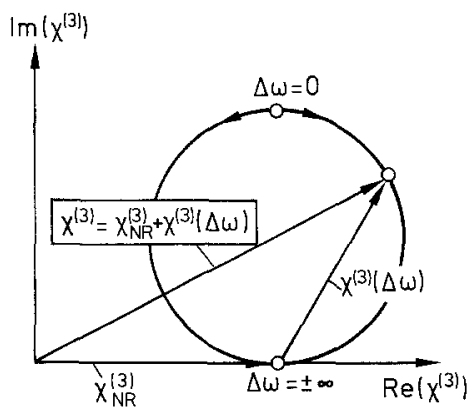

Fig. 1. The complex third-order susceptibility $\chi^{(3)}$ according to (2) in the complex plane. $\Delta \omega=0$ represents the resonance case, and $\Delta \omega= \pm \infty$ the nonresonant situation 
vectors at the various frequencies:

$\Delta k=\left|2 k\left(\omega_{\mathrm{p}}\right)-k\left(\omega_{\mathrm{s}}\right)-k\left(\omega_{\mathrm{as}}\right)\right|$,

$\omega_{\mathrm{as}}=\omega_{\mathrm{p}}+\omega_{\mathrm{R}}=2 \omega_{\mathrm{p}}-\omega_{\mathrm{s}}$

As discussed in detail in [10], the numerical value of $L_{\mathrm{c}}$ can be obtained with great accuracy from the relation $L_{\mathrm{c}}=\lambda_{\mathrm{p}} / D$ with

$D \approx(\Delta v)^{2} \cdot \lambda_{\mathrm{p}}^{4} \cdot d^{2} n(\lambda) / d \lambda^{2}$,

with $\Delta v=\omega_{\mathrm{R}} / 2 \pi c_{0}\left(c_{0}\right.$ the velocity of light $), \lambda_{\mathrm{p}}$ the pump wavelength and $n(\lambda)$ the index of refraction. Since the coherence length depends neither on the magnitude of $n(\lambda)$ nor on its first derivative with respect to wavelength, a precise knowledge of $n(\lambda)$ is necessary to calculate $L_{\mathrm{c}}$, which turns out to be proportional to the curvature of the $n(\lambda)$-dependence and inversely proportional to the Stokes shift $\Delta v$.

\section{Experimental Results}

Nonlinear interferometry requires two successive experiments with phase-controlled superposition of the resultant nonlinear signals. Figure 2 shows experimental details of the collinear CARS configuration. Phase-control of the two signals, labelled with $\omega_{\mathrm{as}}^{\prime}$ and $\omega_{\mathrm{as}}^{\prime \prime}$, can be achieved by use of the phase-shifting unit (P.S.U.). It consists of a pair of adjustable wedges prepared from low-dispersion Schott BK7 glass. A frequency-doubled Nd:YAG laser system produced the pump beam at $\lambda_{\mathrm{p}}=532 \mathrm{~nm}$ and the Stokes beam by excitation of a home-made dye laser. Typical pulse energies were $10 \mathrm{~mJ}$ and $2 \mathrm{~mJ}$ with a pulse duration of $10 \mathrm{~ns}$. Pump and Stokes beams were gently focused $(f=20 \mathrm{~cm})$ into two successive gas cells. Perfect superposition of the two CARS signals could only be achieved if the angular deviation of any optical component between the two signal sources did not exceed 50 seconds of arc and signal detection was restricted to paraxial rays.

For a quantitative comparison between the theoretical prediction of (4) and an actual third-order differencefrequency signal, we took, for preliminary studies, two nonresonant signals produced in methane or butane or two resonant signals of ambient air. Figure 3 shows an interferogram obtained by tuning the dye laser to $\lambda_{\mathrm{s}}=580 \mathrm{~nm}$, corresponding to the excitation of the center of the $Q$-branch $(\Delta J=0, \Delta v=1)$ of atmospheric oxygen with a Stokes shift $\Delta v=1555 \mathrm{~cm}^{-1}$ [11]. Computation of $L_{\mathrm{c}}$ with the available dispersion data for BK-7 glass resulted in $L_{\mathrm{c}}=907 \mu \mathrm{m}$, whereas an experimental value of $910 \mu \mathrm{m}$ was derived from the variation of the wedge thickness. In addition Fig. 3 indicates that such a superposition of two CARS signals permits a convenient calibration of the wedge position in terms of relative phase differences between the two signals. The interferogram also illustrates that the phase position of interferometric minima is an ideal location for low-noise detection of phase-shifted signals.

Next we would like to demonstrate such noisereduction. As resonant signal we took the $S(3)$-line $(\Delta J=2, v=0)$ of 100 Torr of molecular hydrogen with $\Delta v=1035 \mathrm{~cm}^{-1}[12]$. A strong nonresonant background was produced from $4 \mathrm{~atm}$ butane. The left-hand side of Fig. 4 shows the resonant $S(3)$-signal buried in the noise of the nonresonant butane signal. By producing a phasereversed nonresonant signal in the second cell filled with butane only it was possible to completely suppress the nonresonant contribution of the sample prepared from the two gases (right-hand CARS spectrum of Fig. 4).

The availability of two adjustable signals (adjustable in terms of amplitude and phase) allows other signal manipulations in the complex plane, which may be less important for trace analysis, but demonstrate the principle in more detail. Figure 5 shows the sign-reversal of a resonant signal in two steps: starting from a Lorentzian (left-hand side of Fig. 5) with practically no nonresonant background, coherent addition of a $-90^{\circ}$ phase-shifted

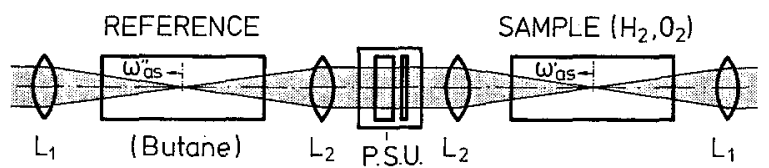

Fig. 2. Experimental arrangement for phase-sensitive addition of two CARS signals produced at locations $\omega_{\mathrm{as}}^{\prime}$ (sample) and $\omega_{\mathrm{as}}^{\prime \prime}$ (reference). P.S.U.: Phase-shifting unit made from a wedge pair of BK-7 prisms of adjustable thickness

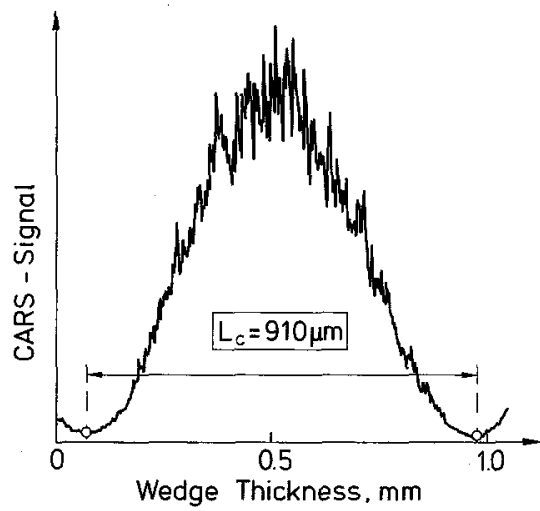

Fig. 3. Determination of the coherence length $L_{\mathrm{c}}$ by variation of the optical pathlength of a pair of BK-7 wedges

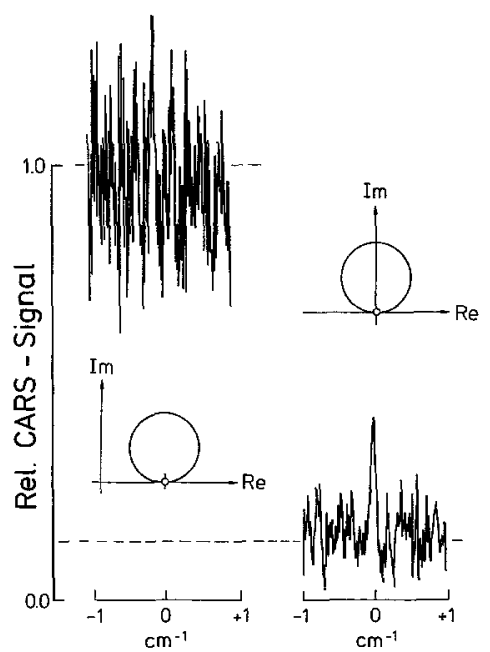

Fig. 4. Nonresonant background suppression and recovery of the resonant $S(3)$ pure rotational signal of $\mathrm{H}_{2}$. Mixture conditions: 100 Torr $\mathrm{H}_{2}$ in $4 \mathrm{~atm}$ of butane 


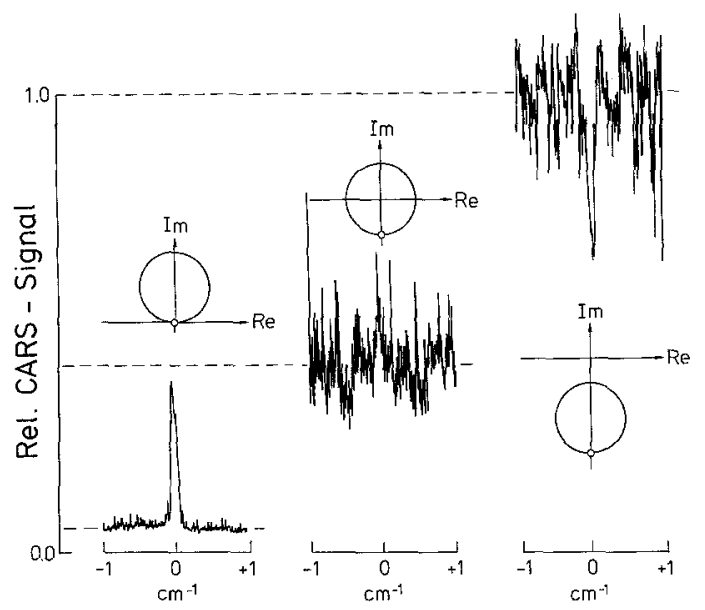

Fig. 5. Line reversal by coherent addition of a phase-reversed nonresonant signal (see text for details)

nonresonant signal of half the amplitude of the resonant signal results in a considerable increase of the total noise level. Finally, addition of a $-90^{\circ}$ phase-shifted nonresonant signal exceeding the amplitude of the resonant signal (right-hand side of Fig. 5) leads again to perfect visibility of the resonant signal with complete sign-reversal.

\section{Discussion}

We have demonstrated that phase-controlled nonlinear interferometry can completely reject the unwanted nonresonant background in a typical CARS configuration. The procedure is superior to the usual polarization-sensitive methods, since it does not decrease the level of the resonant signal under consideration. The identification of the $S(3)$ line of molecular hydrogen under room temperature conditions served as an example. We have also monitored the $S(4)$ line of hydrogen, which is frequently used to analyse the $\mathrm{H}_{2}$-content in high-temperature combustion studies [13]. The successful recovery of the resonant signal strongly depends on the complete cancellation of both nonresonant signals and perfect superposition of the two resonant signals. This requires some experimental expertise but can always be achieved, provided the optical components have the appropriate precision. We have demonstrated the principle for the identification of hydrogen in butane. As shown in more detail in [14], the contribution of any nonresonant signal of unknown origin in practical problems can be performed with respect to phase and amplitude and hence can be completely rejected. The actual degree of background rejection can be derived from Fig. 3: The apparent signal noise levels indicate an increase in detection sensitivity by at least one order of magnitude. Under favorable con- ditions even two orders of actual reduction in concentration of a minority species might be achievable.

Coherent cancellation of background signals was proposed as early as 1980 for liquid samples by Yacoby et al. [15]. They developed a technique that made use of celllength variations and also obtained a reduction in detectability level by one order of magnitude for the analysis of the phenol content in water from $1 \%$ to $0.1 \%$. Our arrangement, using two spatially well separated signal sources and an independent phase-shifting unit, shows more versatility. Experimentally it is slightly easier than the double resonance technique originally proposed by Lynch et al. [16]. An extension of our technique to 3dimensional CARS-configurations is also possible. Such an approach would eliminate another problem that we frequently run into in the course of our experiments: the onset of (nonlinear) saturation effects in collinear configurations [10]. In fact, one might even use the information from an experiment under conditions of coherent (amplitude) superposition to detect and hence exclude saturation effects.

Acknowledgements. The experimental work has been supported by the Deutsche Forschungsgemeinschaft through the Leibniz-Prize program. We thank J. Jethwa for a critical reading of the English version of this manuscript.

\section{References}

1. P.D. Maker, R.W. Terhune: Phys. Rev. 137, A801 (1965)

2. A.C. Eckbreth: "Laser Diagnostics for Combustion Temperature and Species", in Energy \& Engineering Science Series, ed. by A.K. Gupka, D.G. Lilley (Abacus, Tunbridge Wells 1988)

3. H.W. Schrötter, H. Frunder, H. Berger, J.-P. Boquillon, B. Lavorel, G. Millot: in Advances in Non-Linear Spectroscopy (Wiley, New York 1988)

4. T. Kishimoto, N. Wenzel, G. Marowski: CLEO 1989

5. J.-L. Oudar, R.W. Smith, Y.R. Shen: Appl. Phys. Lett. 34, 758 (1979)

6. Y.R. Shen: The Principles of Nonlinear Optics (Wiley, New York 1984)

7. R. Brakel, F.W. Schneider: in Advances in Non-Linear Spectroscopy (Wiley, New York 1988)

8. G. Lüpke, G. Marowsky, R. Steinhoff: Appl. Phys. B 49, 283 (1989)

9. G. Berkovic, Y.R. Shen, G. Marowsky, R. Steinhoff: J. Opt. Soc. Am. B 6, 205 (1989)

10. G. Lüpke: Dissertation, University of Göttingen, 1990

11. T. Dreier, B. Lange, J. Wolfrum, M. Zahn: Appl. Phys. B 45, 183 (1988)

12. U. Fink, T.A. Wiggins, D.H. Rank: J. Mol. Spectrosc. 18, 384 (1965); L. Wolniewicz: J. Chem. Phys. 78, 6173 (1983)

13. A.C. Eckbreth, T.J. Anderson: SPIE 742 (1987) F.Y. Yueh, E.J. Beiting: Appl. Opt. 27, 3233 (1988)

14. G. Marowsky, G. Lüpke, R. Steinhoff, L.F. Chi, D. Möbius: Phys. Rev. B 41, 4480 (1990)

15. Y. Yacoby, R. Fitzgibbon, B. Lax: J. Appl. Phys. 51, 3027 (1980)

16. R.T. Lynch, Jr., S.D. Kramer, H. Lotem, N. Bloembergen: Opt. Commun. 16, $372(1976)$ 